Supporting Information

\title{
Phospholipid and Hydrocarbon Interactions with a Charged Electrode Interface
}

Zachary A. Levine ${ }^{1,2, a}$, Nadica Ivošević DeNardis ${ }^{3, a}$, P. Thomas Vernier*,

1 Department of Physics,

University of California Santa Barbara, Santa Barbara, CA, USA

2 Department of Chemistry and Biochemistry,

University of California Santa Barbara, Santa Barbara, CA, USA

3 Division for Marine and Environmental Research,

Ruđer Bošković Institute, Zagreb, Croatia

$4 \quad$ Frank Reidy Research Center for Bioelectrics,

Old Dominion University, Norfolk, VA, USA

\section{Corresponding author:}

*E-mail: pvernier@odu.edu 


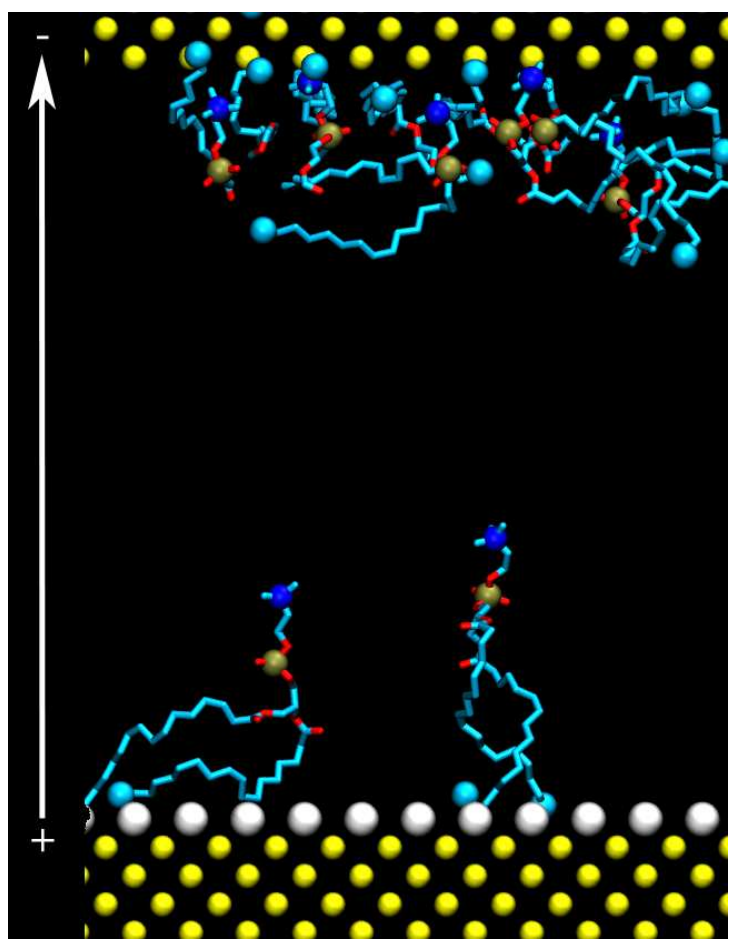

Figure S1. A representative molecular dynamics snapshot showing the behavior of PC molecules near highly charged electrode interfaces. 